Check for updates

Cite this: RSC Adv., 2019, 9, 30698

\title{
Investigations of the charge transfer phenomenon at the hybrid dye/BiVO 4 interface under visible radiation
}

\author{
K. Ordon, ${ }^{a}$ S. Coste, ${ }^{b}$ O. Noel, ${ }^{b}$ A. El-Ghayoury, ${ }^{c}$ A. Ayadi, ${ }^{c}$ A. Kassiba*b \\ and M. Makowska-Janusik (D)*a
}

\begin{abstract}
Photocatalytic hybrid systems were realized by associating bismuth vanadate $\left(\mathrm{BiVO}_{4}\right)$ nanostructured thin films with anchored organic and metal-organic complex molecules. The chosen dyes are based on indoline and azo-based moieties. Optical and photoinduced charge transfer features were investigated experimentally and analysed theoretically through the electron band alignment on the organic/inorganic interface. Quantum calculations were carried out for the studied hybrid systems by using DFT and semiempirical approaches. The calculations were performed by implementing a cluster model applied for the nanostructures and hybrid systems. The electronic density peculiarities point out efficient charge transfer for D149 based hybrids compared to azo-based systems. The electron distribution in hybrid systems inferred from the computational analysis and their experimental probing using Kelvin Force Microscopy (KFM) maps the way to understanding the photoinduced charge transfer occurring at the interfaces between organic dyes and an inorganic photocatalyst. The presented approach helps to predict suitable photoactive hybrid materials leading to efficient photocatalytic devices.
\end{abstract}

Received 13th July 2019

Accepted 20th September 2019

DOI: 10.1039/c9ra05373e

rsc.li/rsc-advances photoinduced charge transfer at the organic-inorganic interfaces. ${ }^{9}$ The main requirement for the hybrid interface assumes low HOMO-LUMO energy difference for the organic molecule and compatibility of its energy levels with respect to those of the semiconducting structure. Thus, an efficient flow of photogenerated electrons occurs from the dyes to the semiconducting material where, in consequence, the number of photoelectrons increases. ${ }^{10}$ In this process, photoactivated electrons are promoted from the dyes HOMO orbital to the conduction band of a semiconductor media where electron-hole $\left(\mathrm{e}^{-}-\mathrm{h}^{+}\right)$pairs are created and move toward the surface of the photocatalyst. ${ }^{\mathbf{1 1}}$ The electronic features of the underlying media define the charge mobility within volume paths and the possible electron-hole recombination time. The $\mathrm{e}^{-}$and $\mathrm{h}^{+}$collected on the surface of the material induce the key oxidation and reduction processes of surface adsorbed species leading to superoxide and hydroxyl radicals on the surrounding media. These processes represent the bases of photocatalytic reactions for water or atmosphere purification from harmful organic pollutants. ${ }^{\mathbf{1 2}}$

The challenging approach developed in the frame of photoactive media for the environment preservation refers to anchored organic dyes at the surface of the oxide photocatalyst. Anchoring dyes on photoactive semiconducting surfaces enhances the light harvesting and then improves the photocatalytic efficiency of the whole hybrid material. In this case, dyes possessing the carboxyl group are popular ${ }^{13-15}$ as those with a catechol group (D- $\pi$-Cat) ensuring the anchoring moiety. ${ }^{\mathbf{1 6}}$
${ }^{a}$ Institute of Physics, Faculty of Mathematics and Natural Science, Jan Dlugosz University in Czestochowa, Al. Armii Krajowej 13/15, 42200 Czestochowa, Poland. E-mail:m.makowska@ujd.edu.pl

${ }^{b}$ Institute of Molecules and Materials, Le Mans University, 72085 Le Mans, France. E-mail: Abdelhadi.Kassiba@univ-lemans.fr

'Laboratoire MOLTECH-Anjou, UMR 6200, CNRS, UNIV Angers, 2 bd Lavoisier, 49045 Angers Cedex, France 
Additionally, these molecules possess a character of the donoracceptor structure $(\mathrm{D}-\pi-\mathrm{A}){ }^{17}$

The present work is devoted to study the association of organic or metalorganic complex dyes with inorganic nanostructured photocatalyst in the aim to enhance the photocatalytic efficiency of the hybrid system as a whole. The investigations deal with exhaustive insights on the charge transfer occurring between the different dye molecules and the inorganic semiconducting nanocompound. The proposed approach combine theoretical modelling supported by numerical simulations and experimental probing of interactions on the dye/semiconductor interface. As a semiconductor, the bismuth vanadate $\left(\mathrm{BiVO}_{4}\right)$ was chosen due to its demonstrated properties as a visible light driven photocatalyst. ${ }^{18,19}$ Two classes of sensitizing organic groups were chosen due to their optical properties. The first organic family consists in D149 dyes ${ }^{20}$ able to be anchored on the inorganic surface via their carboxyl moiety. The second class of molecules possessing the donoracceptor $(\mathrm{D}-\pi-\mathrm{A})$ features is composed by a free ligand and its corresponding zinc(II) complex referred below as LIGAND and COMPLEX, respectively. The COMPLEX and LIGAND were synthetized by our research group as the molecules relevant for optical applications. ${ }^{21}$ We discovered that these molecules absorb efficiently sun light and the idea was to test them for the dye synthetized solar cells or photocatalytic applications. Furthermore, these molecules are considered for the first time as potential charge transfer groups in photocatalytic processes.

The possible effect of hydroxyl radicals on the chemical stability of the azobenzene dyes has been simply tested by observing the colour of the photocatalyst $\left(\mathrm{Dye} / \mathrm{BiVO}_{4}\right)$ solution and measuring its optical absorption spectrum over time. Normally, it is known that the hydroxyl radicals are used in order to treat the wastewater containing azobenzene dyes by their decomposition. In our case, when the experiment was performed, no colour change of the photoactive solution was observed and it may justify the chemical stability of the dyes during the performed photocatalytic experiment.

In order to evaluate the relevance of LIGAND and COMPLEX molecules for the considered applications, commercial dye as D149 was used for comparison. The charge transfer peculiarities were characterized by performing the experimental KFM measurements for COMPLEX/BiVO ${ }_{4}, \mathrm{LIGAND}_{\mathrm{BiVO}}$ and D149/ $\mathrm{BiVO}_{4}$ with and without illumination. These experiments were quantitatively modelled by using quantum chemical calculations applied to the investigated molecules subject to irradiation causing photoinduced charge transfer from dyes to inorganic $\mathrm{BiVO}_{4}$ nanocompound. Thus, D149 dyes anchored on the surface of the $\mathrm{BiVO}_{4}$ allow charge transfer from organic to inorganic compounds. The LIGAND dyes proceed in a similar way as D149 but completely different phenomenon was observed for COMPLEX groups. In consequence, the aim of the presented work is to compare the photoinduced charge transfer performances for the three molecular groups by using suitable optical and KFM experiments supported by theoretical modelling and numerical simulations.

\section{Theoretical background}

Until now, most of the theoretical works related to computational studies of hybrid systems based on semiconductor oxides with anchored charge transfer organic dyes were carried out for $\mathrm{ZnO}^{22}$ or $\mathrm{TiO}_{2}$ (ref. 23) nanostructures. To our best knowledge, the study of charge transfer in hybrid systems based on the $\mathrm{BiVO}_{4}$ nanstructures was not undertaken up to now. The challenging work concerns the electronic properties of the D149/ $\mathrm{BiVO}_{4}, \mathrm{COMPLEX} / \mathrm{BiVO}_{4}, \mathrm{LIGAND} / \mathrm{BiVO}_{4}$ hybrids. Therefore, the cluster model ${ }^{24,25}$ was applied to obtain the frontier orbitals in the inorganic components as well as in the $\mathrm{BiVO}_{4}$ based hybrid materials. In this aim, quantum chemical calculations were performed along with experimental probing of the charge transfer on the hybrid interfaces. The implemented theoretical approach allows better understanding the photoinduced processes occurring in hybrid systems based on $\mathrm{BiVO}_{4}$ used as visible light driven photocatalyst.

The first step was to determine the electronic and optical properties of the D149, LIGAND and COMPLEX molecules (see Fig. 1) as isolated structures simulated in vacuum by using the $a b$ initio formalism implemented in GAMESS program package. ${ }^{26,27}$ The initial geometries were build using ACD/ ChemSketch, the integrated software package from Advanced Chemistry Development, Inc. The minima of the potential energy surface have been calculated at restricted Hartree-Fock (RHF) level with the standard 6-31G basis set in $C_{1}$ symmetry. The RHF SCF calculations were performed with an energy convergence criterion chosen to be $10^{-8}$ hartree. The geometries of molecules were optimized applying the quadratic approximation (QA) of the optimisation algorithm based on augmented Hessian techniques. ${ }^{28}$ The gradient convergence tolerance was equal to $10^{-4}$ hartree per bohr. At the end of a geometry search, the Hessian evaluation was performed to exclude the structures giving rise to negative modes and ensure the thermodynamic stability of the molecule.

Simulations of electronic properties were performed for the optimised structures (see Fig. 1) applying the density functional theory (DFT) implemented in Gaussian program..$^{\mathbf{2 9 , 3 0}}$ The time dependent DFT (TDDFT) calculations were performed using the B3LYP functional according to the results reported in our
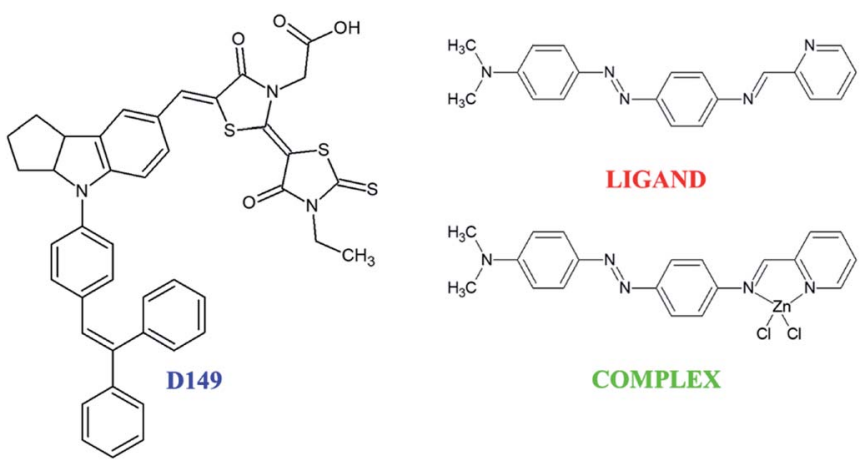

COMPLEX

Fig. 1 Structures of the studied organic dye molecules: D149, LIGAND and COMPLEX. 
previous work. ${ }^{21}$ The single point calculations were performed with $6-31++\mathrm{G}^{* *}$ basis set $^{31}$ and the RHF SCF energy convergence criterion was chosen to be $10^{-12}$ hartree. The UV-vis absorption spectra were calculated using the iterative Davidson method ${ }^{32}$ with an accuracy equal to $10^{-12}$ hartree. The electronic properties of the considered molecules were also calculated using parametrised PM6 semi-empirical methodology at the RHF level applying SCF convergence criterion equal to $10^{-8}$ hartree. The semi-empirical methodology was chosen to compare the data obtained for isolated molecules with those related to hybrid materials based on the $\mathrm{BiVO}_{4}$ nanostructures.

Additionally, it is known that the DFT methodology underestimate the value of a calculated energy difference between frontiers orbitals of molecules. At the same time, it should be noted that the nanostructures in cluster approach are treated as a large-size molecule during quantum chemical calculations. However, the most severe shortcoming of the DFT methods is the self-interaction error. Therefore, the energy of the HOMO orbital does not correspond to the ionization potential and the HOMO-LUMO energy gap splitting ( $\left.\Delta E_{\text {HOMO-LUMO }}\right)$ becomes too small for both polar ionic systems as well. As the nanoparticles exhibit generally polar behaviour, the semiempirical PM6 and PM7 parameterised methods are relevant.

The construction of isolated $\mathrm{BiVO}_{4}$ clusters was realized according to our previous work. ${ }^{24}$ Then, the initial geometries of hybrid configurations were built up with D149, LIGAND and COMPLEX molecules anchored on the surface of the $\left(\mathrm{BiVO}_{4}\right)_{18}$ clusters. Dye molecules were anchored on the surface of the $\left(\mathrm{BiVO}_{4}\right)_{18}$ cluster by vanadium atom. Geometries of the constructed hybrid structures were optimised by using the parameterised PM6 methods with SCF convergence criterion equal to $10^{-8}$ hartree. The gradient convergence tolerance was equal to $10^{-4}$ hartree per bohr. In this case, an organic dye molecule, a linking vanadium atom and three neighbouring oxygen atoms located on the $\mathrm{BiVO}_{4}$ cluster surface serving as grafting sites for the dye molecules were relaxed. The remaining part of the $\mathrm{BiVO}_{4}$ cluster was frozen as it was assumed in our previous work. ${ }^{24}$

The hybrid systems with optimised geometries were used to calculate their electronic and optical properties by using the parameterised PM6 method and the calculations were performed for isolated systems in vacuum using Gaussian program. The SCF convergence criterion was equal to $10^{-8}$ hartree.

\section{Experimental details}

Experimental studies were carried out on porous thin films of monoclinic bismuth vanadate deposited on the borofloat glass according to the procedure described elsewhere. ${ }^{18}$ The hybrid films with anchored dyes were realized according to the following protocol. The 5-[[4-[4-(2,2-diphenylethenyl)phenyl]-1,2,3-3a,4,8bhexahydrocyclopent $[b]$ indol-7-yl] methylene]-2-(3-ethyl-4-oxo-2-thioxo-5-thiazolidinylidene)-4-oxo-3-thiazolidineacetic acid (D149) was purchased from Sigma-Aldrich and used without further purification. The other substances defined as $N, N$-dimethyl-4-((E((pyridin-2-ylmethylene)amino)phenyl)diazenyl)aniline (LIGAND) and $\quad N, N$-dimethyl-4-((E(-(pyridin-2-ylmethylene)amino)phenyl) diazenyl)aniline zinc chloride (COMPLEX) were synthesized in MOLTECH-Anjou Laboratory according to a previous report. ${ }^{21}$ All other solvents as tert-buthanol or acetonitrile were purchased from Merck and Sigma-Aldrich. The sensitizing procedures consist in using $3.34 \mathrm{mg}$ of the D149, $4.8 \mathrm{mg}$ of the LIGAND or $1 \mathrm{mg}$ of the COMPLEX dissolved in mixture of $7.5 \mathrm{ml}$ acetonitrile and $7.5 \mathrm{ml}$ tert-butanol. To completely dissolve the organic dye powders, the solutions were submitted to ultra-sonification and heated at $66^{\circ} \mathrm{C}$ for $30 \mathrm{~min}$. After completing the dissolution process, the solutions were cooled down at room temperature. Then, the mesoporous thin films were immersed in the dye solutions $\left(0.0003 \mathrm{~mol} \mathrm{l}^{-1}\right)$ during $4 \mathrm{~h}$ to ensure efficient adsorption of dyes on $\mathrm{BiVO}_{4}$ surface. The optical properties were investigated by using Ocean Optics UVvis spectrophotometer in the wavelength range from $190 \mathrm{~nm}$ to $1100 \mathrm{~nm}$. The surface features of the hybrid films were characterized by using a Bruker DI 3100 (Nanoscope V) atomic force microscope. The morphology of the thin films and their roughness were determined using the tapping mode ${ }^{\mathrm{TM}}$ AFM. Based on the AFM setup, KFM measurements were performed to map the surface electrical potential of the film and its evolution from dark to light exposure with or without anchored dyes. For the illumination, the optical fiber integrated in the AFM setup was used. The samples were illuminated by a white light. Morphology of the hybrid mesoporous films and their electrical activity were probed by using a two-pass KFM technique (Bruker DI3100-Nanoscope V controller). All measurements have been done with the same AFM probe coated by platine-iridium layer required for optimal electrical properties. The nominal cantilever constant was $3 \mathrm{~N} \mathrm{~m}^{-1}$. The measurements were done line by line to probe the topography of the profile in a first step, and then by passing over the same profile for measuring the contact potential difference (CPD) at a given separation distance between the probe and the sample. Therefore, the measurements were realized by using a two-pass mode. Prior to the experiments, three AFM probes were selected by doing force curve and KFM measurements on a referenced silicon wafer. Images were taken after one pass over the sample to be sure that the illumination of the sample was efficient.

\section{Results and discussion}

\section{Theoretical approach and simulation}

Organic dye molecules. The DFT/B3LYP and the semiempirical parameterised PM6 method were applied to compute the electronic properties of D149, LIGAND and COMPLEX molecules. The semi-empirical approach allows a pertinent evaluation of the physical features of systems with large sizes and with the involvement of heavy atoms. The method is also relevant for the calculations of electronic properties of hybrids.

The energy values calculated for the frontier orbitals of isolated molecules and their energy splitting $\Delta E_{\text {Hомо-Lumo }}$ are presented in Table 1 . The $\Delta E_{\text {HOMO-Lumo values calculated by }}$ DFT/B3LYP method for all three molecules correlate with the optical absorption measured experimentally. ${ }^{21}$ With regard to the criterion of energy levels at the interfaces, the performed calculations suggest that the D149 and LIGAND dyes are appropriate for sensitization of the $\mathrm{BiVO}_{4}$ clusters as inferred 
Table 1 Energy of the HOMO and LUMO orbitals, $\Delta E_{\text {HOMO-LUMO }}$ and dipole moments of the D149, LIGAND and COMPLEX molecules calculated by DFT/B3LYP and PM6 method

\begin{tabular}{|c|c|c|c|c|c|c|}
\hline & DFT/B3LYP & PM6 & DFT/B3LYP & PM6 & DFT/B3LYP & PM6 \\
\hline LUMO $[\mathrm{eV}]$ & -2.55 & -1.54 & -2.11 & -0.95 & -3.30 & -1.92 \\
\hline$\Delta E_{\text {HOMO-LUMO }}[\mathrm{eV}]$ & 2.58 & 6.65 & 2.91 & 6.91 & 2.17 & 5.78 \\
\hline$\mu[\mathrm{D}]$ & 12.22 & 10.37 & 4.28 & 3.91 & 13.70 & 12.54 \\
\hline
\end{tabular}

from the reduction potential of the monoclinic $\mathrm{BiVO}_{4}$ equal to $4.5 \mathrm{eV}^{33}$ Additionally, analysing the data collected in Table 1, underlines that the LUMO energy of D149 and LIGAND molecules is higher than the reduction potential of the considered semiconductor in contrast to the LUMO level of the COMPLEX, which is lower. On the other hand, as shown from the performed calculation of a charge distribution, the D149 and COMPLEX molecules exhibit a high polarity compared to the LIGAND groups. The results presented in Table 1 show that the PM6 method overestimates the calculated $\Delta E_{\text {номо-Luмо value }}$ but the general tendency of the electronic properties changes obtained by the DFT calculations is preserved.

The features of the HOMO and LUMO orbitals for the D149, LIGAND and COMPLEX molecules calculated by the DFT/B3LYP and PM6 methods do not show any significant differences and the frontier orbitals obtained by the semiempirical approach are presented in Fig. 2. The НОМO orbital of the D149 molecule is mainly located on the donor part of the molecule (from biphenyl up to indoline group) while the LUMO orbital is more shifted close to the carboxyl group. It suggests that the HOMO to LUMO transition represents important charge separation. Therefore, it can confirm that an intramolecular charge transfer (ICT) occurs when the HOMO-LUMO transition is induced. It is also supported by a significant dipole moment of the molecule. The HOMO orbitals of the LIGAND and COMPLEX molecules are located on the dimethylamino group possessing donor

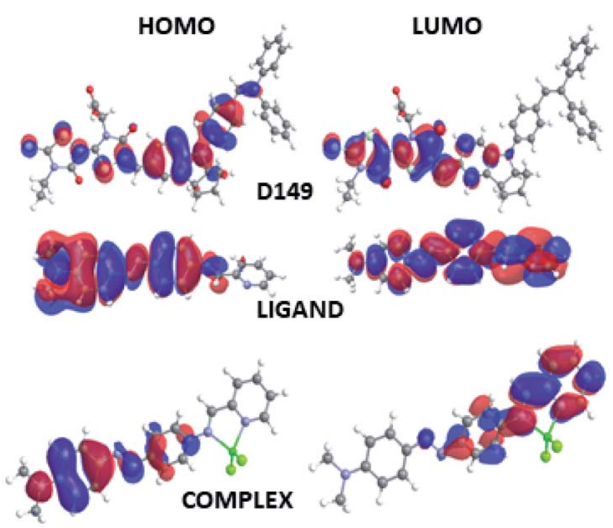

Fig. 2 The HOMO and LUMO orbitals of the D149, LIGAND and COMPLEX dye molecules calculated by the semi-empirical PM6 parameterized method. character. The iminopyridine group plays the role of acceptor where the LUMO orbitals are predominantly located. Comparing the frontiers orbitals distribution occurring in LIGAND and COMPLEX molecules one may see that they are more separated in the COMPLEX structure. It means that the HOMO to LUMO electron transition creates significant charge separation in COMPLEX molecule. In addition, the HOMO and LUMO orbitals indicate a stronger donoracceptor behaviour in the COMPLEX. This is most likely caused by the Lewis acid character of the $\mathrm{ZnCl}_{2}$ fragment. ${ }^{21}$ It gives also impact at the dipole moment of these two molecules. The dipole moment of the COMPLEX is higher than the dipole moment of LIGAND. Concluding, one may say that in all the studied cases the ICT may occurs qualifying the selected molecules as dyes.

$\left(\mathrm{BiVO}_{4}\right)_{18}$ cluster. The electronic properties of the $\left(\mathrm{BiVO}_{4}\right)_{18}$ cluster were calculated using the semi-empirical PM6 parametrised method. The obtained data were analysed in details in our previous work ${ }^{24}$ and underline that the HOMO and LUMO orbitals are located in the surface part of the cluster. The analysis of the frontier orbitals distribution shows significant similarities to the partial electron density of states calculated for the bulk $\mathrm{BiVO}_{4}$ material. The HOMO orbitals can be compared to a valence band from where photogenerated electrons flow, while the LUMO orbitals can be assimilated to the conduction band. The VBM of the $\mathrm{BiVO}_{4}$ crystal structure are built from $\mathrm{O} 2 \mathrm{p}$ orbitals slightly hybridized with $\mathrm{Bi}$ 6s states. Typically in metal oxides the valence band is derived from $\mathrm{O} 2 \mathrm{p}$ orbitals and the conduction band is built by metal $\mathrm{s}$ or $\mathrm{d}$ orbitals. In monoclinic $\mathrm{BiVO}_{4}$ its CBM is dominated by the $\mathrm{V}$ $3 \mathrm{~d}$ states with small contributions from $\mathrm{Bi} 6 \mathrm{p}$ and $\mathrm{O} 2 \mathrm{p}$ orbitals. The partial DOS computed for partially reconstructed $\left(\mathrm{BiVO}_{4}\right)_{18}$ cluster indicates that the highest occupied energy levels are associated mainly to the $\mathrm{O} 2 \mathrm{p}$ states hybridized with $\mathrm{Bi} 6 \mathrm{p.}{ }^{24}$ The LUMO orbital of the $\left(\mathrm{BiVO}_{4}\right)_{18}$ cluster is composed by V $3 \mathrm{~d}$ states with small contributions from $\mathrm{Bi} 6 \mathrm{p}$ and $\mathrm{O} 2 \mathrm{p}$ states as it was also observed for the bulk system. One could conclude that the size reduction of the investigated cluster does not drastically change its electronic features. The $\Delta E_{\text {номо-цuмо }}$ difference calculated for the $\left(\mathrm{BiVO}_{4}\right)_{18}$ by semi-empirical PM6 parametrised method is equal to $2.40 \mathrm{eV} .{ }^{24}$ Analysing data obtained for the LUMO orbital of the $\left(\mathrm{BiVO}_{4}\right)_{18}$ cluster one may conclude that the vanadium atoms are the most appropriate to be involved into molecular bonding between dye and the inorganic nanostructure. 


\section{Theoretical approach for hybrid systems}

Based on the analysis of the LUMO orbitals in the studied dyes, the $-\mathrm{COOH}$ and iminopyridine groups were chosen in D149, LIGAND as well as in the COMPLEX molecules, respectively. The carboxyl and iminopyridine groups are the moieties of the molecules where a high electron density, forming the LUMO orbital, is located. Hybrid systems were built combining organic molecules via selected bonding groups with $\left(\mathrm{BiVO}_{4}\right)_{18}$ cluster. The D149/BiVO 4 hybrid system was realized by anchoring the carboxyl group of the D149 molecule to the vanadium atom located on the surface of the nanocluster. For COMPLEX/BiVO and LIGAND/BiVO 4 systems, the connection occurs through the nitrogen atom of the iminopyridine group. In order to preserve a thermodynamic equilibrium of the hybrid system its geometry was optimised according to the total energy minimization process described in the paragraph Theoretical background. As a consequence, the hybrid structure based on $\left(\mathrm{BiVO}_{4}\right)_{18}$ cluster was stable with the possible lowest total energy.

Electronic properties of the investigated hybrid systems referred below as $\mathrm{HD}$ for $\mathrm{D} 149 /\left(\mathrm{BiVO}_{4}\right)_{18}$, HL for LIGAND/ $\left(\mathrm{BiVO}_{4}\right)_{18}$ and $\mathrm{HC}$ for COMPLEX/(BiVO $)_{18}$ were calculated by PM6 method and the obtained results are summarized in Table 2. The PM6 method was chosen due to its relevance to predict the results compared to the experimental structural and electronic data of $\mathrm{BiVO}_{4}$ clusters $^{24}$ The anchored D149 molecule lowers the $\Delta E_{\mathrm{HOMO}}$ LuMo compared to the pure semiconducting cluster. Oppositely, the COMPLEX group increase the energy gap of the $\left(\mathrm{BiVO}_{4}\right)_{18}$ cluster while the LIGAND groups did not modify the $\Delta E_{\mathrm{HOMO}}$ LuMO of the sensitized $\mathrm{BiVO}_{4}$ cluster. The $\Delta E_{\text {HOMO-LuMO }}$ calculated for the $\left(\mathrm{BiVO}_{4}\right)_{18}$ cluster by using the parametrised PM6 semiempirical method is equal to $2.40 \mathrm{eV}^{24}$ The obtained results point out the relevance of the D149 dyes to sensitize the $\mathrm{BiVO}_{4}$ nanostructures in contrast to the LIGAND or COMPLEX. It may be supposed because the D149 dye anchored on the surface of the $\left(\mathrm{BiVO}_{4}\right)_{18}$ cluster decreases the energy gap of the nanostructure.

Such conclusion is also supported by the shape of the frontier orbitals inferred from calculations on the optimized hybrid structures as depicted in Fig. 3. In the case of HD hybrid system the HOMO is located at the interface between dye and nanocluster. Its LUMO orbital is localized on the semiconducting nanostructure. The observed localization of the HOMO and LUMO orbitals leads to improved photoinduced charge transfer from the D149 molecule to the $\mathrm{BiVO}_{4}$ cluster able to enhance the photocatalytic properties of the considered systems.

Table 2 Energy of the HOMO and LUMO orbitals, $\triangle E_{\text {HOMO-LUMO }}$ and dipole moments of the $\left(\mathrm{BiVO}_{4}\right)_{18} / \mathrm{D} 149(\mathrm{HD}),\left(\mathrm{BiVO}_{4}\right)_{18} / \mathrm{LIGAND}(\mathrm{HL})$ and $\left(\mathrm{BiVO}_{4}\right)_{18} / \mathrm{COMPLEX}(\mathrm{HC})$ hybrid systems calculated by semiempirical PM6 parametrised method

\begin{tabular}{lrrr}
\hline & HD & \multicolumn{1}{c}{ HL } & \multicolumn{1}{c}{ HC } \\
\hline HOMO [eV] & -4.30 & -5.06 & -5.00 \\
LUMO [eV] & -2.19 & -2.62 & -1.66 \\
$\Delta E_{\text {HOMO-LUMO }}[\mathrm{eV}]$ & 2.11 & 2.43 & 3.34 \\
$\mu[\mathrm{D}]$ & 21.94 & 36.09 & 29.54
\end{tabular}

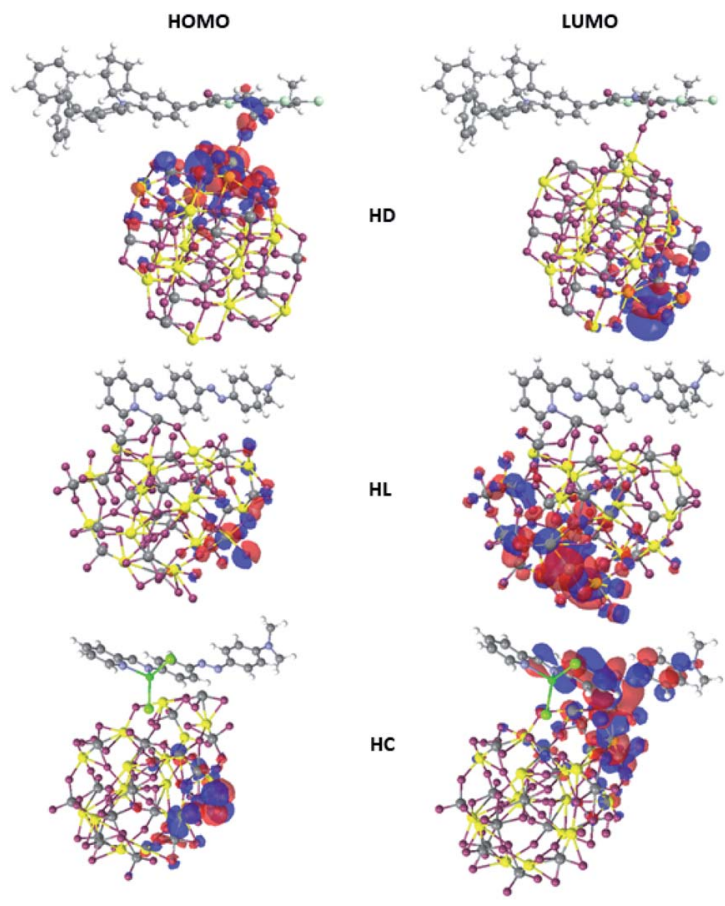

Fig. $3 \mathrm{HOMO}$ and LUMO orbitals of the D149, LIGAND and COMPLEX dye molecules calculated by the semi-empirical PM6 parameterized method.

In the case of HL system, both the HOMO and LUMO orbitals are localized at the $\mathrm{BiVO}_{4}$ cluster. It means that the anchored dyes will not induce significant effect on the charge transfer across the hybrid system. In this case, the dyes do not participate to the process of the photo-activated charge flow between the dyes and the nanostructure. Significant difference is seen for the $\mathrm{HC}$ system, where a photoactivated charge transfer occurs from the inorganic cluster to the organic dye. It is a completely different charge flow that is theoretically ordered. In this case, the HOMO orbital is located at the semiconducting nanoparticle but the LUMO is distributed at the COMPLEX molecule. Therefore, the COMPLEX molecule works as an inhibitor in photoactivated processes. Thus, the data summarized in Fig. 3 indicate that the D149 molecule is the most appropriate for the charge transfer from the organic group to the inorganic semiconducting $\mathrm{BiVO}_{4}$ nanocluster.

\section{Optical characterization}

Optical properties of dyes. In order to support the calculations of the electronic properties of dye molecules, the theoretical optical absorption spectra were compared to the experimental ones. The spectra were simulated for isolated dyes applying semi-empirical PM6 parametrised method and the results are presented in Fig. $4 \mathrm{a}$.

The UV-vis absorption spectra were prepared using Gabedit program. Taking into account the oscillator strength, the calculated absorption lines were convoluted using Gaussian line shape where the half-width was equal to $20 \mathrm{~nm}$.

The UV-vis absorption spectra were also measured for D149, LIGAND and COMPLEX molecules dissolved in the acetonitrile/ 

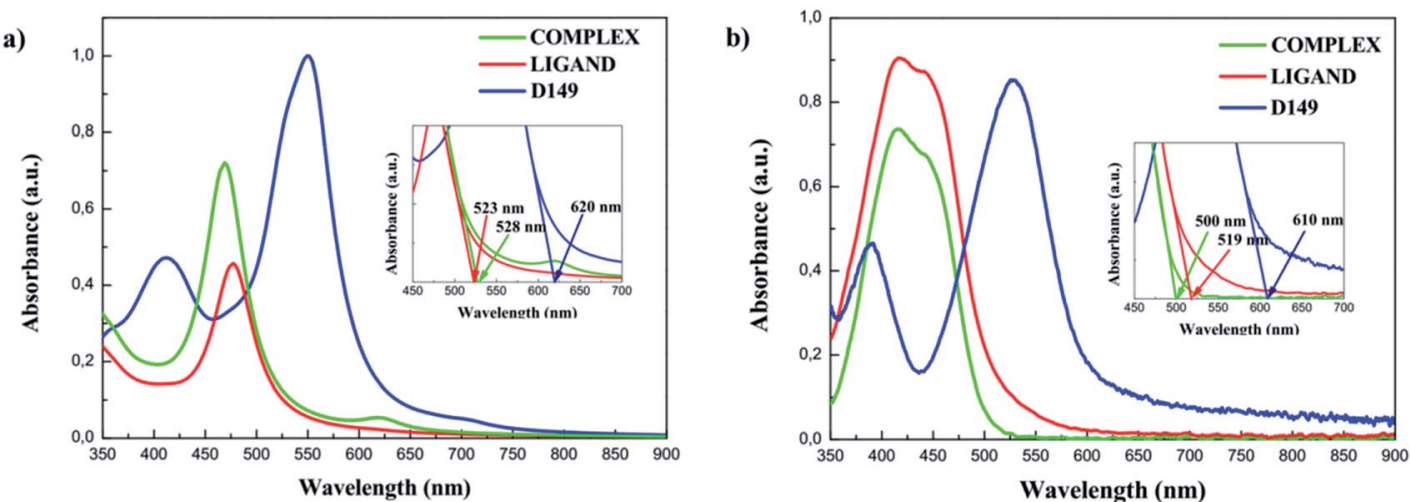

Fig. 4 UV-vis absorption spectra of the COMPLEX, LIGAND and D149 molecules calculated by semi-empirical PM6 parametrized method (a) and measured experimentally for the same molecules dissolved in acetonitrile/tert-butanol (1/1) mixture (b).

tert-butanol as $1 / 1$ volume ratio and the results are presented in Fig. 4b. The D149 dyes dissolved in the acetonitrile/tert-butanol exhibits strong absorption around $530 \mathrm{~nm}$, i.e. close to the maximum irradiance of the solar spectrum. ${ }^{34}$ The semi-empirical PM6 parametrised method reproduces well optical properties of the D149 molecule. The LIGAND molecules studied experimentally (see Fig. 4b) exhibits strong electronic absorption band at $438 \mathrm{~nm}$ which is assigned to the $\mathrm{n} \rightarrow \pi^{*}$ absorption bands of the azobenzene moiety and the pyridyl ring together with a slight intramolecular charge transfer (ICT) from the dimethylamino donor group to the iminopyridine accepting unit. ${ }^{21,35}$ The quantum chemical calculations (see Fig. 4a) reproduce well the nature of the UV-vis absorption spectrum of the LIGAND. However, the spectrum is shifted towards long wavelength.

The UV-vis absorption spectrum measured for the COMPLEX molecules shows a bathochromic shift of the first absorption peak compared to the LIGAND molecule. It can be explained by an increase of the electron acceptor behaviour of the COMPLEX upon zinc complexation. The absorption peak located at $450 \mathrm{~nm}$ in the theoretical spectrum of the COMPLEX molecule is compatible with the first absorption peak observed in the experiment. Calculations of the UV-vis absorption spectrum for the COMPLEX molecules account also the small shoulder

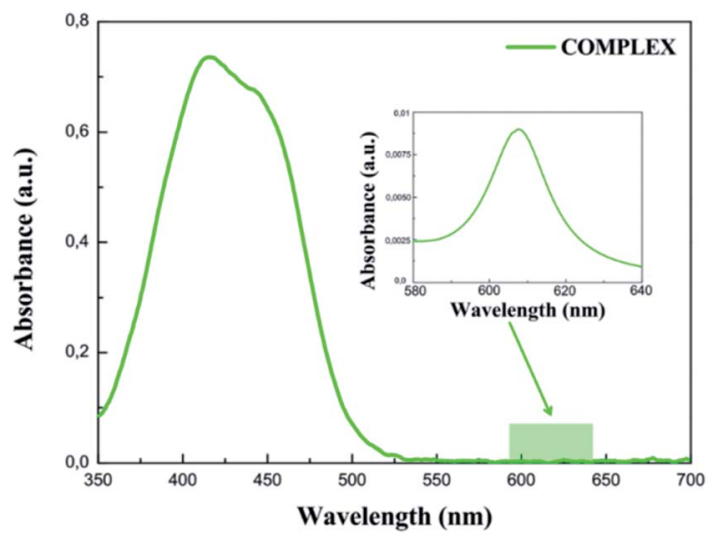

Fig. 5 Experimental UV-vis absorption spectrum measured for the COMPLEX molecule dissolved in acetonitrile/tert-butanol (1/1). observed experimentally at $610 \mathrm{~nm}$ (Fig. 5). This peak is observed when the chelation of iminopyridine fragment to the zinc(II) ion is important. ${ }^{21}$ The experimental UV-vis absorption spectrum measured for the COMPLEX molecules dissolved in acetonitrile/tert-butanol (1/1) mixture is presented in Fig. 5. One may see that the intensity of the absorption peak at the $610 \mathrm{~nm}$ depends on the chelation level of the iminopyridine moiety of the molecules.

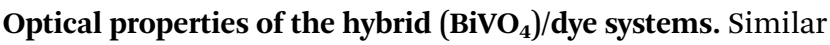
optical characterization was carried out on hybrid systems and the results are summarized in Fig. 6. The UV-vis absorption spectrum measured for HD system exhibits an increased absorption background up to $650 \mathrm{~nm}$. The measured spectrum is composed by the output of the inorganic thin film and the absorption band related to the used organic dye (see Fig. 6a). The broad UV-vis absorption band measured for HD system is located between $480 \mathrm{~nm}$ and $630 \mathrm{~nm}$. The UV-vis absorption spectra did not match with superposition of respective absorption bands of organic groups and inorganic $\mathrm{BiVO}_{4}$. An
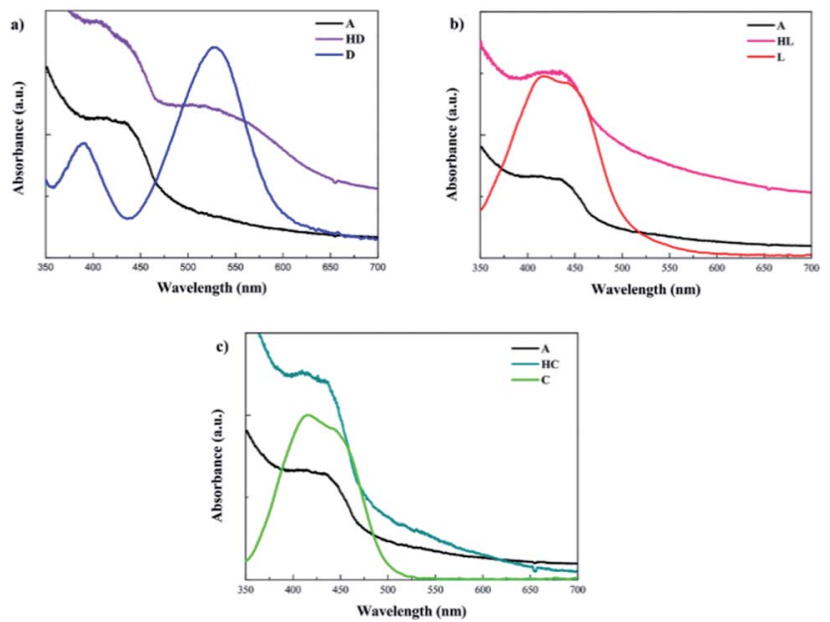

Fig. 6 UV-vis absorption spectra measured for $\mathrm{BiVO}_{4}$ thin film (A), organic dyes D149 (D), ligand (L) and complex (C), and their hybrid systems HD (a), HL (b) and HC (c) dissolved in the acetonitrile/tertbutanol (1/1). 
additional broad background is noticed on the optical absorption of hybrid systems, which we attribute to charge transfer effects. It is probably due to the interaction between the molecular orbitals of the dye with the $\mathrm{BiVO}_{4}$ electron structure. On may conclude that the D149 dye anchored to the $\mathrm{BiVO}_{4}$ surface extends its absorption capabilities in the visible range compared to the pure semiconducting structure. The appearance of the above mentioned absorption band illustrates the influence of the dye on the sensitized network and traduces the involvement of charge transfer band as it was described by Hori et $a$. $^{36}$

The UV-vis absorption spectra were also measured for $\mathrm{HL}$ and HC systems. An analysis of the data presented in Fig. $6 \mathrm{~b}$ and $c$ indicates that the effect of the LIGAND and COMPLEX dyes is not as significant as it is noticed for the HD hybrid system. COMPLEX or LIGAND groups anchored to the $\mathrm{BiVO}_{4}$ nanocluster alters in minor extent the absorption spectrum. The change has not reached the limit of absorption, which is entirely in line with the absorption of the $\mathrm{BiVO}_{4}$ bare film and no additional charge transfer band was observed. This allows us to conclude that LIGAND as well as COMPLEX dyes are no longer relevant as sensitizing elements for $\mathrm{BiVO}_{4}$ photocatalyst.

\section{KFM studies}

The AFM and KFM measurements were performed for the $\mathrm{BiVO}_{4}$ thin films with anchored D149, LIGAND and COMPLEX molecules. The AFM tip used for the measurements has a platine-iridium coated to ensure good electrical conductive properties and the nominal cantilever constant is $3 \mathrm{~N} \mathrm{~m}^{-1}$. Topographic and contact potential difference (CPD) images have been obtained by using the same AFM tip. To ensure that no contamination occurs during the measurements, force curve and CPD images have been regularly recorded on a reference silicon wafer surface. Measurements without illumination were obtained by checking that the laser light reflected on the cantilever is located at the center of the cantilever and that no interferences occur in between the sample and the cantilever. Thus, the laser light intensity spreading on the sample surface may be negligible. Measurements with illumination were obtained by lighting the sample using the optical fiber of the AFM. This optical fiber is independent of the laser/cantilever system and it directly illuminates the sample at an angle without interfering with the laser light.

In the Fig. 7a a surface topologies of the $\mathrm{BiVO}_{4}$ structures with anchored dyes are presented. The AFM images shows that the sensitized mesoporous films exhibit grain sizes larger compared to the pure $\mathrm{BiVO}_{4}$ films. This illustrates significant importance of the anchored species on film surface increasing its effective thickness. In addition, the analysis of the KFM measurements showed a net effect from the dye molecules on the surface electrical potential distribution illustrated on an area about $1 \mu \mathrm{m} \times 1 \mu \mathrm{m}$. The Fig. 7b shows the KFM surface potential distribution without irradiation by visible light and the Fig. 7c illustrates the situation under irradiation. The characteristic phenomenon occurring in this case consists in blurring the surface potential, especially under visible radiation

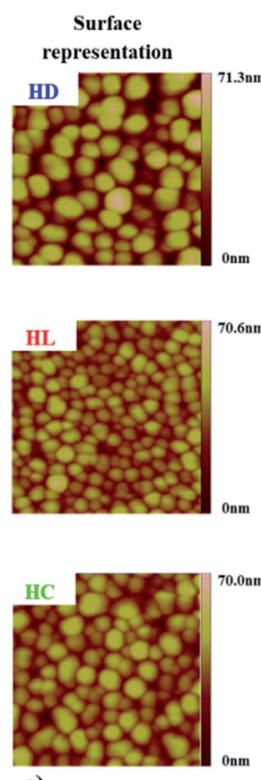

a)
Surface potential
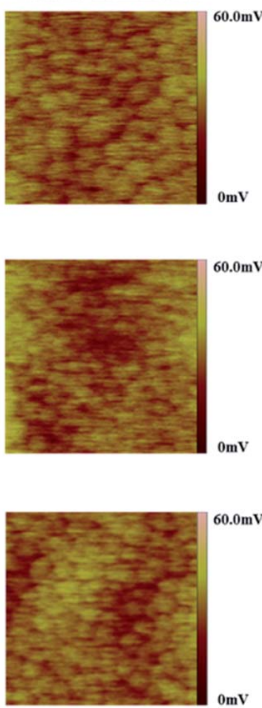

b) without illumination
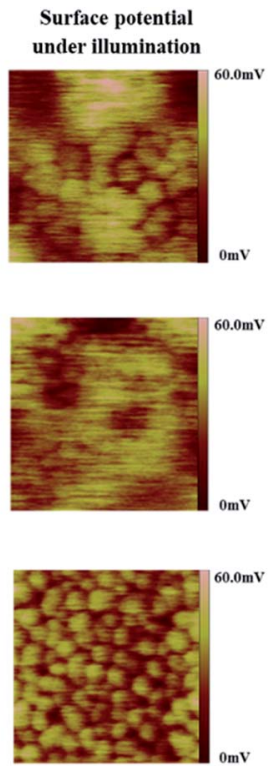

c)
Fig. 7 Surface topologies of the $\mathrm{BiVO}_{4}$ structures with anchored D149 $(\mathrm{HD})$, ligand $(\mathrm{HL})$ and complex $(\mathrm{HC})$ dyes measured by AFM (a) and their KFM surface potential distribution recorded without irradiation (b) and under visible light illumination (c).

exposure. For the non-irradiated samples (Fig. 7b), the highest potential is focused on the individual particles and corresponds closely to the surface topography of the film. At the opposite, under irradiation, the potential is blurred and does not correlate directly with the surface morphology (Fig. 7c) as clearly shown for the HD and HL systems.

The resulting behaviour for the hybrid systems is explained by the efficiency of charge transfer between the constitutive elements. Electrons excited from the HOMO to LUMO levels of the organic molecules can be then transferred to the conduction band of the semiconductor. The increased amount of charge carriers at the interfaces leads to a free flow of surface charges. In the case of the HC system, the situation is different than it was observed for the HD and HL hybrids. The effect of the irradiation causes the reverse charge transfer reaction exhibiting the potential located at the individual $\mathrm{BiVO}_{4}$ nanoparticles. These experimental data are in good agreement with the expected behaviour from theoretical predictions and underline a weak photoactive compatibility between the $\mathrm{BiVO}_{4}$ thin film and the COMPLEX molecule.

The problem of charge transfer can be analysed in the general framework of the electronic band alignment and Fermi level pinning as it occurs in hetero-junctions with acceptor (A)donor (D) model (see Fig. 8). As discussed in our former work ${ }^{\mathbf{1 1}}$ on the $\mathrm{BiVO}_{4}$ sensitized by the D149, the process of the charge transfer mediated by the photon absorption by $\mathrm{D}$ groups causes a shift of the Fermi energy. It can occur when difference of a surface potentials exist at the dye $/ \mathrm{BiVO}_{4}$ interface. The KFM investigations performed for the dye/ $/ \mathrm{BiVO}_{4}$ system probe the surface potential, which is modified under light irradiation. The difference between the work function of the inorganic layer and 

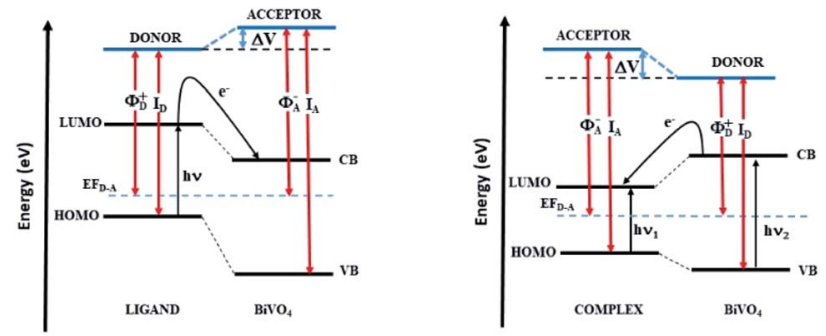

Fig. 8 Electronic band alignment at the hybrid interfaces.

ionization energy of the organic groups correlate with the electrical potential change probed by KFM technique. Based on the electronic band alignment and the experimental optical absorption spectra for the considered dyes we expect optimal charge transfer for the $\mathrm{D} 149 / \mathrm{BiVO}_{4}$ and less effects for LIGAND/ $\mathrm{BiVO}_{4}$ interfaces due to higher HOMO-LUMO energy but a striking behaviour was noticed for COMPLEX/BiVO

As results of our study, we compare the energy diagrams for the LIGAND $/ \mathrm{BiVO}_{4}$ and COMPLEX/BiVO ${ }_{4}$ systems (Fig. 8). They were plotted in the same convention as it was done for the D149/ BiVO $_{4}{ }^{11}$ The presented diagram convinces that in the LIGAND/ $\mathrm{BiVO}_{4}$ systems under illumination the charge is transferred from dye to the semiconducting nanostructure as it is also noticed for the $\mathrm{D} 149 / \mathrm{BiVO}_{4}$ hybrid. In the COMPLEX/BiVO system the excited nanostructure populates the dye levels. In this case, the CB of the $\mathrm{BiVO}_{4}$ is located above the LUMO of the COMPLEX molecule. This situation is not appropriate for photocatalytic applications planed on such hybrid systems.

The described transfer model between the organic molecule and the photocatalyst is based on the treatment of the hybrid system as the tandem of the two components, as described in the work of Hara and coauthors. ${ }^{37,38}$ The nature of the interaction at the interfaces has been investigated for photovoltaic systems and described as persistent chemical bonding. ${ }^{39,40}$ In this case, the main reason of the difference between surface potential in virgin and sensitized hybrids may be related to the work function and its modification by the electrical activities induced by the anchored dyes.

The performed studies point out the behaviour of photoinduced electrical activity on the $\mathrm{BiVO}_{4}$ as function of the irradiation and the involvement of anchored dyes able to facilitate charge transfer from organic dyes to the $\mathrm{BiVO}_{4}$ cluster through the hybrid interface.

\section{Conclusions}

Based on a combined strategy of computational and experimental investigations of the dye/ $/ \mathrm{BiVO}_{4}$ hybrid systems, the charge transfer between the dye molecules and the nanocrystals has been demonstrated. As efficient hybrid system, the $\mathrm{BiVO}_{4}$ sensitized by the D149 molecules exhibits compatible electron band alignment for efficient charge transfer at the hybrid interfaces. In the case of the $\mathrm{BiVO}_{4}$ sensitized by LIGAND and COMPLEX molecules, no significant changes in electronic or optical properties have been observed in relation to the bare $\mathrm{BiVO}_{4}$ thin films.

The analysis using AFM instrumentation in connection with the investigations of the surface potential distribution (KFM) of mesoporous $\mathrm{BiVO}_{4}$ with anchored organic dye molecules confirms the results obtained by applying theoretical analysis of the electron density distribution on the frontier orbitals. The investigations were carried out for the $\mathrm{BiVO}_{4}$ thin film samples with anchored D149, LIGAND and COMPLEX molecules before and during light irradiation. For the HD and HL samples, the surface potential under the additional irradiation was blurred, while for the HC sample the reverse phenomenon was observed. In this case, the potential is focused on the particles of the material as it was confirmed from the frontier orbitals representation supported by the KFM measurements.

The optical properties of the investigated hybrid systems were described. The UV-vis absorption spectrum measured for the HD system shows additional broad background that can be related to prominent charge transfer band between the sensitizing organic molecules and the underlying inorganic support. In the opposite, anchored LIGAND and COMPLEX molecules do not create any additional absorption band due to the peculiarities of the HUMO-LUMO orbitals of the organic molecules compared to the band features of the $\mathrm{BiVO}_{4}$ cluster. The photoactivity of the investigated systems is under consideration for environmental issues.

\section{Conflicts of interest}

There are no conflicts of interest to declare.

\section{Acknowledgements}

Calculations have been carried out in Wroclaw Center of Networking and Supercomputing (Grant no. 171). K. Ordon gratefully acknowledges the financial support given by Excellent EIFFEL Grant from French Government and Doctoral School 3MPL - Le Mans University. K. Ordon is grateful to the NCN Polish Science National Centre for financial support under grant Preludium No. 2016/21/N/ST3/00455.

\section{Notes and references}

1 D. Bahnemann, Sol. Energy, 2004, 77, 445.

2 R. Andreozzi, V. Caprio, A. Insola and R. Marotta, Catal. Today, 1999, 53, 51.

3 I. P. Parkin and R. G. Palgrave, J. Mater. Chem., 2005, 15, 1689.

4 P. Liska, K. R. Thampi, M. Grätzel, D. B. D. Rudmann, H. M. Upadhyaya and A. N. Tiwari, Appl. Phys. Lett., 2006, 88, 203103.

5 H. C. Wu, Y. C. Peng and T.-P. Shen, Materials, 2012, 5, 2088. 6 R. Asahi, T. Morikawa, T. Ohwaki, K. Aoki and Y. Taga, Science, 2001, 293, 269.

7 G. Li, D. Zhang and J. C. Yu, Chem. Mater., 2008, 20, 3983.

8 A. V. Vorontsov, Catal. Today, 2015, 252, 168. 
9 V. C. Anitha, A. N. Banerjee and S. W. Joo, J. Mater. Sci., 2015, 50, 7495.

10 J. Chae, D. Young Kim, S. Kim and M. J. Kang, Ind. Eng. Chem., 2010, 16, 906.

11 K. Ordon, V. I. Merupo, S. Coste, O. Noel, N. Errien, M. Makowska-Janusik and A. Kassiba, Appl. Nanosci., 2018, 8, 1895.

12 J.-M. Herrmann, Catal. Today, 1999, 53, 115.

13 H. Ozawa, T. Sugiura, R. Shimizu and H. Arakawa, Inorg. Chem., 2014, 53, 9375.

14 F. De Angelis, S. Fantacci and A. Selloni, Nanotechnology, 2008, 19, 424002.

15 C. I. Oprea, P. Panait, F. Cimpoesu, M. Ferbinteanu and M. A. Gîrțu, Materials, 2013, 6, 2372.

16 Y. Ooyama, T. Yamada, T. Fujita, Y. Harimaa and J. J. Ohshita, J. Mater. Chem. A, 2014, 2, 8500.

17 Y. Ooyama, Y. Shimada, S. Inoue, T. Nagano, Y. Fujikawa, K. Komaguchi, I. Imae and Y. Harima, New J. Chem., 2011, 35, 111.

18 R. Venkatesan, S. Velumani, K. Ordon, M. MakowskaJanusik, G. Corbel and A. Kassiba, Mater. Chem. Phys., 2018, 205, 325.

19 V. I. Merupo, S. Velumani, K. Ordon, N. Errien, J. Szade and A. Kassiba, CrystEngComm, 2015, 17, 3366.

20 H.-M. Cheng, Nanotechnology, 2010, 21, 485202.

21 I. Guezguez, A. Ayadi, K. Ordon, K. Iliopoulos, D. Branzea, A. Migalska-Zalas, M. Makowska-Janusik, A. El-Ghayoury and B. Sahraoui, J. Phys. Chem. C, 2014, 118, 7545.

22 M. Habib, N. N. Ghosh, R. Sarkar, A. Pramanik, P. Sarkar and S. Pal, Chem. Phys. Lett., 2018, 709, 21.

23 M. Zalas, B. Gierczyk, A. Bossi, P. R. Mussini, M. Klein, R. Pankiewicz, M. Makowska-Janusik, Ł. Popenda and W. Stampor, Dyes Pigm., 2018, 150, 335.

24 K. Ordon, A. Kassiba and M. Makowska-Janusik, RSC Adv., 2016, 6, 110695.

25 M. A. Ruiz Preciado, A. Kassiba, A. Morales-Acevedo and M. Makowska-Janusik, RSC Adv., 2015, 5, 17396.
26 M. S. Gordon and M. W. Schmidt, Theory Appl. Comput. Chem.: First Forty Years, 2005, 1167.

27 M. W. Schmidt, K. K. Baldrige, J. A. Boatz, S. T. Elbert, M. S. Gordon, J. H. Jensen, S. Koseki, N. Matsunaga, K. A. Nguyen, S. J. Su, et al., J. Comput. Chem., 1993, 14, 1347. 28 F. J. Jensen, Chem. Phys., 1995, 102, 6706.

29 J. B. Froresman and A. E. Frisch, Exploring Chemistry with Electronic Structure Methods, Gaussian, Inc., U. S. A., 2nd edn, 1993.

30 M. J. Frisch, G. W. Trucks, H. B. Schlegel, G. E. Scuseria, M. A. Robb, J. R. Cheeseman, G. Scalmani, V. Barone, B. Mennucci and G. A. Petersson, et al., Gaussian 09, Gaussian, Inc., Wallingford CT, 2009.

31 T. Clark, J. Chandrasekhar, G. W. Spitznagel and P. Schleyer, J. Comput. Chem., 1983, 4, 294.

32 E. R. Davidson, J. Comp. Physiol., 1975, 17, 87.

33 U. Habib, A. A. Tahir and T. K. Mallick, Appl. Catal., B, 2018, 224, 895.

34 E. Guillen, J. Idıgoras, T. Berger, J. A. Anta, C. FernandezLorenzo, R. Alcantara, J. Navasb and J. Martın-Calleja, Phys. Chem. Chem. Phys., 2011, 13, 207.

35 K. G. Yager and C. J. Barrett, Amorphous azobenzene polymers for light-induced surface patterning, in Smart Light-Responsive Materials, ed. Y. Zhao and T. Ikeda, John Wiley \& Sons, Inc., 2009.

36 T. Hori, H. Moritou, N. Fukuoka, J. Sakamoto, A. Fujii and M. Ozaki, Materials, 2010, 3, 4915.

37 K. Hara, M. Kurashige, Y. Danoh, C. Kasada, A. Shinpo, S. Suga, K. Sayama and H. Arakawa, New J. Chem., 2003, 27, 783.

38 J. M. Macak, M. Zlamal, J. Krysa and P. Schmuk, Small, 2007, 3,300 .

39 T. Hannappel, B. Burfeindt, W. Storck and F. J. Willig, J. Phys. Chem. B, 1997, 101, 6799.

40 F. Sauvage, D. Chen, P. Comte, F. Huang, L.-P. Heiniger, Y.-B. Cheng, R. A. Caruso and M. Grätzel, ACS Nano, 2010, 4, 4420 . 